\title{
@creative
}

\section{On the number of additive permutations and Skolem-type sequences*}

\author{
Diane M. Donovan \\ Centre for Discrete Mathematics and Computing, University of Queensland, \\ St. Lucia 4072, Australia \\ Michael J. Grannell ${ }^{\dagger}$ \\ School of Mathematics and Statistics, The Open University, \\ Walton Hall, Milton Keynes MK7 6AA, United Kingdom
}

Received 3 May 2016, accepted 31 May 2017, published online 9 November 2017

\begin{abstract}
Cavenagh and Wanless recently proved that, for sufficiently large odd $n$, the number of transversals in the Latin square formed from the addition table for integers modulo $n$ is greater than $(3.246)^{n}$. We adapt their proof to show that for sufficiently large $t$ the number of additive permutations on $[-t, t]$ is greater than $(3.246)^{2 t+1}$ and we go on to derive some much improved lower bounds on the numbers of Skolem-type sequences. For example, it is shown that for sufficiently large $t \equiv 0$ or $3(\bmod 4)$, the number of split Skolem sequences of order $n=7 t+3$ is greater than $(3.246)^{6 t+3}$. This compares with the previous best bound of $2^{\lfloor n / 3\rfloor}$.
\end{abstract}

Keywords: Additive permutation, Skolem sequence, transversal.

Math. Subj. Class.: 05B07, 05B10

\section{Introduction}

This paper is concerned with counting additive permutations and Skolem-type sequences. Additive permutations are related to certain kinds of transversals in Latin squares. A Latin square of order $n$ may be envisaged as an $n \times n$ array having $n$ distinct entries, each of which appears once in any one row and once in any one column. We adopt a slightly wider

\footnotetext{
*We thank the referees for helpful comments and for drawing to our attention the papers [6, 7, 8].

$\dagger$ Corresponding author.

E-mail addresses: dmd@maths.uq.edu.au (Diane M. Donovan), m.j.grannell@open.ac.uk (Michael J. Grannell)
} 
than usual definition of a transversal in an $n \times n$ array (not necessarily a Latin square) as a set of $n$ (row, column, entry) triples that cover every row, every column and $n$ distinct entries from the array. Consequently, a transversal in a Latin square will necessarily cover every entry precisely once.

In a recent paper, Cavenagh and Wanless [3] proved that, for all sufficiently large odd $n$, the number of transversals in the Latin square formed from the addition table for integers modulo $n$ is greater than $(3.246)^{n}$. This result was a substantial improvement on previous results in this area, although Vardi [10] conjectured that this number exceeds $c^{n} n$ ! for some constant $c \in(0,1)$. A proof of this conjecture is claimed in the arXiv paper [6]. However, it is important to note that not all transversals are suitable for our purposes.

For integers $a$ and $b$ with $a<b$ we use the notation $[a, b]$ to denote the set of integers $i$ such that $a \leq i \leq b$. An additive permutation $\pi$ on $[-t, t]$ is a permutation of these integers such that $\{i+\pi(i): i \in[-t, t]\}$ is also a permutation on the same set of integers. This definition of an additive permutation is the one employed by Abram [1] and others in connection with Skolem sequences, but the reader is cautioned that it differs from that used in [6] where pointwise addition of permutations on $[1, n]$ is taken modulo $n$. An examination of the proof given in [3] shows that it is possible to adapt the proof to show that the number of additive permutations on $[-t, t]$ is greater than $(3.246)^{2 t+1}$ for all sufficiently large $t$, and this is done in Theorem 2.1 below. There are strong connections between additive permutations and Skolem-type sequences. We investigate some of these connections and obtain much improved lower bounds on the numbers of some Skolem-type sequences.

A pure Skolem sequence, sometimes simply called a Skolem sequence, of order $n$ is a sequence $\left(s_{1}, s_{2}, \ldots, s_{2 n}\right)$ of $2 n$ integers satisfying the following conditions.

(C1) For each $k \in\{1,2, \ldots, n\}$ there are precisely two elements of the sequence, say $s_{i}$ and $s_{j}$, such that $s_{i}=s_{j}=k$.

(C2) If $s_{i}=s_{j}=k$ and $i<j$ then $j-i=k$.

For example, $(4,1,1,5,4,2,3,2,5,3)$ is a pure Skolem sequence of order 5. It is well known that a pure Skolem sequence of order $n$ exists if and only if $n \equiv 0$ or $1(\bmod 4)$. For this and other existence results mentioned below see, for example, [4, 5].

An extended Skolem sequence of order $n$ is a sequence $\left(s_{1}, s_{2}, \ldots, s_{2 n+1}\right)$ of $2 n+1$ integers satisfying $(\mathrm{C} 1)$ and $(\mathrm{C} 2)$ above and such that precisely one element of the sequence is zero. An extended Skolem sequence of order $n$ exists for every positive integer $n$. If the zero element of an extended Skolem sequence of order $n$ appears in the $2 n$-th position, i.e. $s_{2 n}=0$, then the sequence is called a hooked Skolem sequence. A hooked Skolem sequence of order $n$ exists if and only if $n \equiv 2$ or $3(\bmod 4)$. If the zero element of an extended Skolem sequence of order $n$ appears in the $(n+1)$-th position, i.e. $s_{n+1}=0$, then the sequence is called a split Skolem sequence or a Rosa sequence. A split Skolem sequence of order $n$ exists if and only if $n \equiv 0$ or $3(\bmod 4)$.

A split-hooked Skolem sequence (also known as a hooked Rosa sequence) of order $n$ is a sequence $\left(s_{1}, s_{2}, \ldots, s_{2 n+2}\right)$ of $2 n+2$ integers satisfying (C1) and (C2) above and such that $s_{n+1}=s_{2 n+1}=0$. A split-hooked Skolem sequence of order $n$ exists if and only if $n \equiv 1$ or $2(\bmod 4)$ and $n \neq 1$.

The various types of Skolem sequence described above may be used to construct solutions to Heffter's first and second difference problems. These, in turn, may be used to construct cyclic Steiner triple systems. We will refer to the sequences just described, and 
to some near relatives, somewhat loosely as Skolem-type sequences. It was shown in $[1,2]$ that for many of these Skolem-type sequences (in particular, pure, hooked, split and splithooked) the number of them is essentially bounded below by $2^{\left\lfloor\frac{n}{3}\right\rfloor}$, where $n$ is the order of the sequence.

\section{Additive permutations}

Define the Latin square $A_{n}$ of odd order $n=2 t+1$ to have its rows and columns indexed by the integers in $[-t, t]$ and the $(i, j)$ entry $k \in[-t, t]$ given by $k \equiv i+j(\bmod n)$. The array $A_{n}$ gives the addition table on $\mathbb{Z}_{n} . \mathrm{A} \mathbb{Z}$-transversal $T$ in $A_{n}$ is a transversal in which every (row, column, entry) triple $(i, j, k) \in T$ has $k=i+j$ in $\mathbb{Z}$, so that no triples of a $\mathbb{Z}$-transversal have $i+j<-t$ or $i+j>t$. Not all transversals in $A_{n}$ are $\mathbb{Z}$-transversals, for example the transversal formed by the leading diagonal in $A_{n}$ is not a $\mathbb{Z}$-transversal. We will only count $\mathbb{Z}$-transversals: in effect the $(i, j)$ cells in $A_{n}$ where $i+j<-t$ or $i+j>t$ are ignored. The entries in these cells are therefore irrelevant to our discussions and it will sometimes be helpful to take $i+j$ as the entry, rather than $i+j$ reduced modulo $n$. This has the advantage that the "ignored" cells are easily identified, particularly when considering subarrays of $A_{n}$ - such cells are then precisely those with entries outside the range $[-t, t]$.

Figure 1 shows the array $A_{19}$ with the "ignored" entries greyed-out, and with a $\mathbb{Z}$ transversal having its entries marked in boxes. For the present, disregard the highlighting of the subarrays.

If the (row, column, entry) triples of a $\mathbb{Z}$-transversal in $A_{n}$ are listed as a $3 \times n$ array $T^{*}$ with row numbers of $A_{n}$ forming the first row of $T^{*}$, column numbers the second, and entries the third, then each row of this array contains the integers $[-t, t]$, and the entries in the third row are the sums of the corresponding entries in the other two rows. Taking the first row of $T^{*}$ as $[-t, t]$, the second row as a permutation $\pi$ on $[-t, t]$, and the third row as the vector sum of the first two rows, then $\pi$ is an additive permutation on $[-t, t]$. Conversely, if $\pi$ is an additive permutation on $[-t, t]$ then the (row, column, entry) triples $(i, \pi(i), i+\pi(i))$ for $i \in[-t, t]$ form a $\mathbb{Z}$-transversal in $A_{n}$. If the entries in the third row of such a $3 \times n$ array $T^{*}$ are multiplied by $(-1)$, then each column of the resulting array sums to zero, and the new array is called a zero-sum array. Thus, there is an equivalence between $\mathbb{Z}$-transversals, additive permutations, and zero-sum arrays. Table 1 (taken from [3]) gives the number of these, here denoted by $z_{n}$, for $n=2 t+1 \leq 23$. These numbers form the sequence A002047 in Sloane's encyclopaedia [9] and have been independently checked by ourselves using our own computer program. The table also gives a rounded down value for $\left(z_{n}\right)^{\frac{1}{n}}$ which will be used subsequently. We remark that $z_{2 t+1}$ is also the number of extremal Langford sequences with defect $t+1$ (i.e. starting with $t+1$ ) - see $[1,4]$ for definitions.

Theorem 2.1. Suppose that $b$ and $n$ are odd and that $n \geq 3 b \geq 9$. Then $z_{n} \geq\left(z_{b}\right)^{2\left\lfloor\frac{n-b}{2 b}\right\rfloor}$.

Proof. Our proof is a re-working of that of Cavenagh and Wanless [3], ensuring that for general $b$ and $n$ the subarrays $R$ and $S$ (defined below) have appropriate (sub-)transversals and that the transversals constructed in $A_{n}$ are indeed $\mathbb{Z}$-transversals. We take $b=2 a+1$ (so that $a \geq 1$ ) and $n=2 t+1$. Put $k=\left\lfloor\frac{n-b}{2 b}\right\rfloor=\left\lfloor\frac{t-a}{b}\right\rfloor, s=t-a-b k$, and $r=s+b$. Then $0 \leq s<b, b \leq r<2 b$, and one of $r, s$ is odd and the other is even.

Next define the subarray $M_{(i, j), c}$ of $A_{n}$ to be the $c \times c$ block whose top left entry is in 


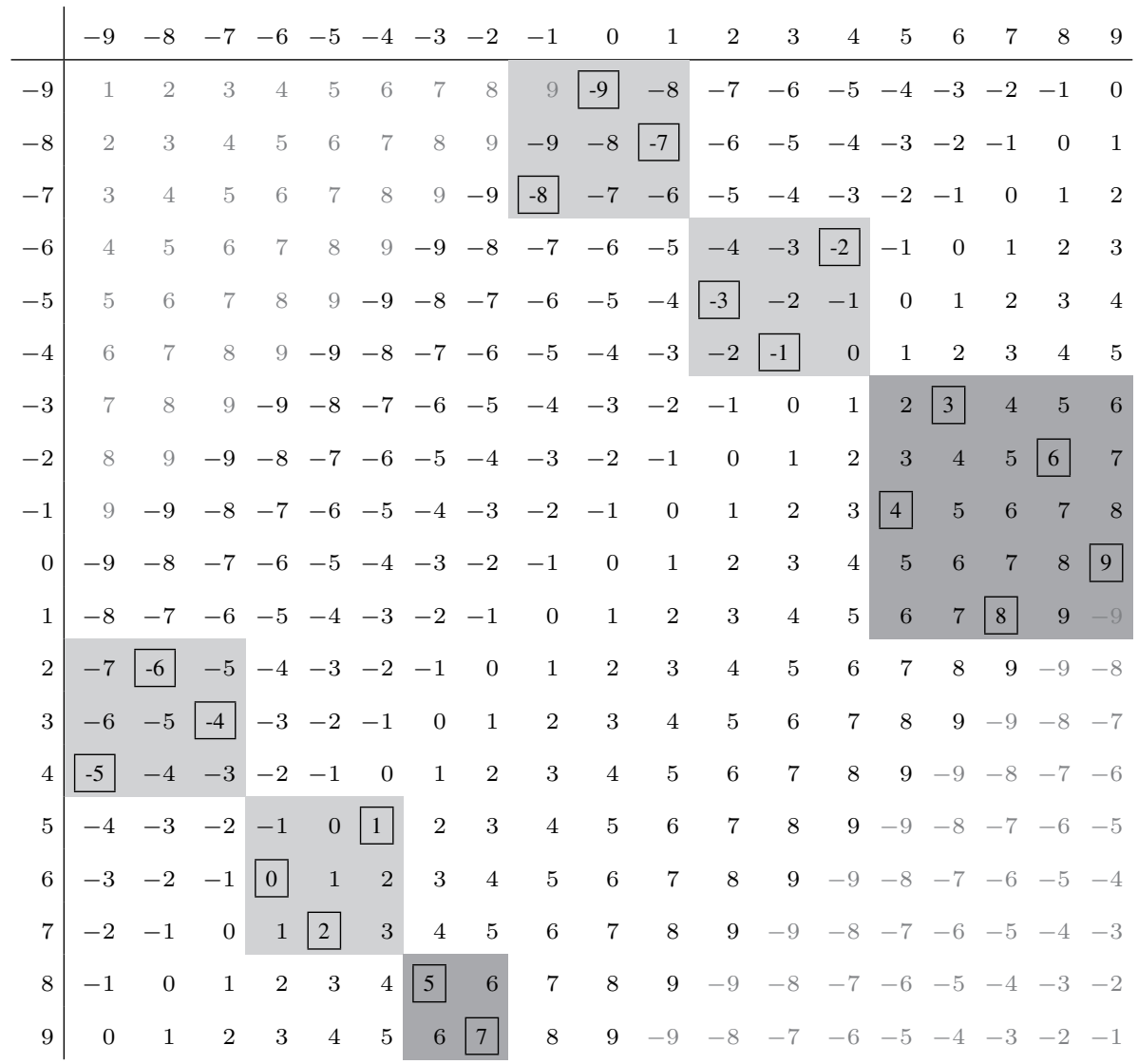

Figure 1: The Latin square $A_{19}$.

Table 1: The number of $\mathbb{Z}$-transversals in $A_{n}$.

\begin{tabular}{r|r|r}
$n$ & $z_{n}$ & $\left(z_{n}\right)^{\frac{1}{n}}>$ \\
\hline \hline 3 & 2 & 1.259 \\
5 & 6 & 1.430 \\
7 & 28 & 1.609 \\
9 & 244 & 1.841 \\
11 & 2544 & 2.039 \\
13 & 35600 & 2.239 \\
15 & 659632 & 2.443 \\
17 & 15106128 & 2.644 \\
19 & 425802176 & 2.845 \\
21 & 14409526080 & 3.046 \\
23 & 577386122880 & 3.246
\end{tabular}


position $(i, j)$ of the array $A_{n}$. For example, if $n=19$ (see Figure 1) then

$$
M_{(-6,2), 3}=\left(\begin{array}{rrr}
-4 & -3 & -2 \\
-3 & -2 & -1 \\
-2 & -1 & 0
\end{array}\right)
$$

As previously mentioned, it is convenient to take the entries of subarrays unreduced modulo $n$. Allowing this, $M_{(i, j), c}$ has entries from $i+j$ to $i+j+2(c-1)$ and, if $c$ is odd, there is a central entry $i+j+c-1$. We use the following subarrays:

Type 1: $D_{i}=M_{(-t+i b,-a+i b), b}$ for $i=0,1, \ldots,(k-1)$,

Type 2: $E_{i}=M_{(-t+r+(k+i) b,-t+i b), b}$ for $i=0,1, \ldots,(k-1)$,

Type 3: $R=M_{(-t+k b,-a+k b), r}$ and

Type 4: $S=M_{(t-s+1,-a-s), s}$.

In Figure $1, n=19$ and $b=3$, so that $t=9, a=1, k=2, s=2$ and $r=5$. The subarrays of types 1 and 2 are lightly shaded and the subarrays $R$ and $S$ are shaded more heavily.

Altogether there are $2 k+2$ subarrays of the four types. No two of these have a common row and the total number of rows covered is $2 b k+r+s=n$, so each row of $A_{n}$ is covered by precisely one of these subarrays. Similarly, each column of $A_{n}$ is covered by precisely one of these subarrays. Consequently we may attempt to construct $\mathbb{Z}$-transversals in $A_{n}$ from transversals in the subarrays.

The type 1 subarray $D_{i}$ has a central entry $2 i b-t+a$. If this value is subtracted from every entry in $D_{i}$, the resulting array is a copy of $A_{b}$. Since $A_{b}$ has $z_{b} \mathbb{Z}$-transversals, each $D_{i}$ has $z_{b}$ transversals that are symmetric about its central entry, that is to say transversals each covering the entries from $2 i b-t$ to $2 i b-t+(b-1)$ inclusive. Note that these transversals avoid "ignored" cells of $A_{n}$. Similarly, the type 2 subarray $E_{i}$ has $z_{b}$ transversals symmetric about its central entry $2 i b-t+3 a+1$, and each of these covers the entries from $2 i b-t+b$ to $2 i b-t+2 b-1$ inclusive. Collectively the type 1 and type 2 subarrays have $\left(z_{b}\right)^{2 k}$ transversals covering the entries from $-t$ to $t-r-s$ inclusive. All of these are partial $\mathbb{Z}$-transversals of $A_{n}$.

To complete the proof for all $b \geq 3$ and $n \geq 3 b$ we must show that the remaining subarrays $R$ and $S$ have appropriate transversals (i.e. avoiding "ignored" cells of $A_{n}$ ) that cover the entry values from $t-r-s+1$ to $t$ inclusive. It will then follow that $A_{n}$ has at least $\left(z_{b}\right)^{2 k}=\left(z_{b}\right)^{2\left\lfloor\frac{n-b}{2 b}\right\rfloor} \mathbb{Z}$-transversals.

The subarray $R$ has entries ranging from $t-r-s-a+1$ to $t+a$ inclusive, and so $R$ always contains some "ignored" cells of $A_{n}$, namely those with entries exceeding $t$. The subarray $S$ has entries ranging from $t-r-s+2+a$ to $t-a-1$ inclusive, so $S$ does not contain any "ignored" cells of $A_{n}$. If we subtract $t-s-a$ from all the entries in $R$ and $S$ we obtain equivalent arrays $R^{\prime}$ and $S^{\prime}$, where $R^{\prime}$ has entries ranging from $-(s+2 a)$ to $s+2 a$, while $S^{\prime}$ has entries ranging from $-(s-1)$ to $s-1$, and we are seeking transversals in these arrays that cover the entry values from $-(s+a)$ to $s+a$ inclusive. Cells in $R^{\prime}$ that contain entries greater than $s+a$ correspond to the "ignored" cells of $R$. Our proof that such transversals always exist falls into a number of cases, the details of which are lengthy, and so are postponed until Section 4.

Corollary 2.2. If $n$ is odd and sufficiently large, then $z_{n}>(3.246)^{n}$. 
Proof. Theorem 2.1 gives $z_{n} \geq\left(z_{b}\right)^{2\left\lfloor\frac{n-b}{2 b}\right\rfloor} \geq\left(z_{b}\right)^{\frac{n}{b}-3}$ for $b$ odd and all sufficiently large $n$. From Table 1 we have $\left(z_{23}\right)^{\frac{1}{23}}>3.246$, so taking $b=23$ we obtain $z_{n}>(3.246)^{n}$ for all sufficiently large $n$.

Putting the corollary into words, the number of additive permutations on $[-t, t]$ is greater than $(3.246)^{2 t+1}$ for all sufficiently large $t$.

\section{Skolem-type sequences}

A connection between additive permutations and Skolem-type sequences is formed by socalled $(m, 3, c)$-systems. A set $\mathcal{D}=\left\{D_{1}, D_{2}, \ldots, D_{m}\right\}$, where each $D_{i}$ is a triple of positive integers $\left(a_{i}, b_{i}, a_{i}+b_{i}\right)$ with $a_{i}<b_{i}$ and $\bigcup_{i=1}^{m} D_{i}=\{c, c+1, \ldots, c+3 m-1\}$ is called an $(m, 3, c)$-system. As remarked in [1], such a system exists if and only if

(i) $m \geq 2 c-1$, and

(ii) $m \equiv 0$ or $1(\bmod 4)$ if $c$ is odd, or $m \equiv 0$ or $3(\bmod 4)$ if $c$ is even.

Given an $(m, 3, c)$-system $\mathcal{D}=\left\{D_{1}, D_{2}, \ldots, D_{m}\right\}$, where $D_{i}=\left(a_{i}, b_{i}, a_{i}+b_{i}\right)$, and putting $r=c+3 m-1$, a (Skolem-type) sequence $\left(x_{-r}, x_{-r+1}, \ldots, x_{r-1}, x_{r}\right)$ may be constructed by putting $x_{-\left(a_{i}+b_{i}\right)}=a_{i}, x_{-b_{i}}=a_{i}, x_{-a_{i}}=a_{i}+b_{i}, x_{a_{i}}=b_{i}, x_{b_{i}}=a_{i}+b_{i}$, $x_{a_{i}+b_{i}}=b_{i}$ for $i=1,2, \ldots, m$, and $x_{j}=0$ for $-c<j<c$. For example, if $c=2$ and $m=3$, and if $\mathcal{D}=\left\{D_{1}, D_{2}, D_{3}\right\}$ where $D_{1}=(2,6,8), D_{2}=(3,7,10), D_{3}=(4,5,9)$ then the constructed sequence is

$$
(3,4,2,3,2,4,9,10,8,0,0,0,6,7,5,9,8,10,6,5,7) .
$$

Observe that in such a sequence, for each $k \in\{c, c+1, \ldots, r\}$ the two positions occupied by $k$ are precisely $k$ apart. Further observe that, independently for each $i \in\{1,2, \ldots, m\}$, we may replace $x_{j}$ for $j \in\left\{-a_{i}-b_{i},-b_{i},-a_{i}, a_{i}, b_{i}, a_{i}+b_{i}\right\}$ by $x_{j}^{\prime}$ where $x_{-\left(a_{i}+b_{i}\right)}^{\prime}=b_{i}$, $x_{-b_{i}}^{\prime}=a_{i}+b_{i}, x_{-a_{i}}^{\prime}=b_{i}, x_{a_{i}}^{\prime}=a_{i}+b_{i}, x_{b_{i}}^{\prime}=a_{i}, x_{a_{i}+b_{i}}^{\prime}=a_{i}$. Thus we may obtain $2^{m}$ distinct sequences of length $2 r+1$ each of which has the property that for each $k \in\{c, c+1, \ldots, r\}$, the two positions occupied by $k$ are precisely $k$ apart. Each such sequence has zeros in the central $2 c-1$ positions.

If $\pi$ is an additive permutation on $[-t, t]$ then a $(2 t+1,3, t+1)$-system is formed by the set of triples $\left\{D_{i}: i \in[-t, t]\right\}$ where

$$
D_{i}=(i+2 t+1, \pi(i)+4 t+2, i+\pi(i)+6 t+3) .
$$

Note that the first entries in these triples cover the interval $[t+1,3 t+1]$, the second entries cover $[3 t+2,5 t+2]$, and the third entries cover $[5 t+3,7 t+3]$. If $\pi_{1}$ and $\pi_{2}$ are two different additive permutations on $[-t, t]$, then the two resulting $(2 t+1,3, t+1)$-systems contain different triples. Consequently the number of different $(2 t+1,3, t+1)$-systems is bounded below by $z_{2 t+1}$, and hence by $(3.246)^{2 t+1}$ for all sufficiently large $t$. Combining this with the previous observation that each such sequence gives rise to $2^{2 t+1}$ Skolem-type sequences, we obtain the following result.

Theorem 3.1. For all sufficiently large $t$, there are more than $(6.492)^{2 t+1}$ Skolem-type sequences of length $14 t+7$ having the following properties:

(a) there are zeros in the central $2 t+1$ positions, and 
(b) for $k \in\{t+1, t+2, \ldots, 7 t+3\}$, the two positions occupied by $k$ are precisely $k$ apart.

If the central $2 t+1$ zero entries in such a Skolem-type sequence are replaced by a split Skolem sequence of order $t$ (which exists for $t \equiv 0$ or $3(\bmod 4))$ then a split Skolem sequence of order $7 t+3$ is obtained. Hence we have the corollary:

Corollary 3.2. For sufficiently large $t \equiv 0$ or $3(\bmod 4)$, there are more than $(6.492)^{2 t+1}$ split Skolem sequences of order $7 t+3$.

In fact we can achieve slightly better than this because the number of split Skolem sequences of order $t$ is at least $2^{\left\lfloor\frac{t}{3}\right\rfloor}$ for all $t \equiv 0$ or $3(\bmod 4)[1,2]$, so we have at least that number of choices for replacing the central zeros. The bound $(6.492)^{2 t+1}>2^{5.3973 t}$ is (for large $t$ ) substantially better than the previous best bound of $2^{\left\lfloor\frac{7 t+3}{3}\right\rfloor}$.

Given a split Skolem sequence of order $n$, we can form a pure Skolem sequence of order $n+1$ by replacing the central zero with $n+1$ and placing a further entry $n+1$ at either the start or the end of the sequence. Hence we obtain:

Corollary 3.3. For sufficiently large $t \equiv 0$ or $3(\bmod 4)$, there are more than $(6.492)^{2 t+1}$ pure Skolem sequences of order $7 t+4$.

In the next two corollaries, the lower bound of $(6.492)^{2 t+1}$ is extended to hooked and split-hooked Skolem sequences of orders $7 t+4$ and $7 t+5$ respectively. In each case the basic approach is as follows. For given $t$, choose a small positive integer $c$ such that $t-c \equiv 2$ or $11(\bmod 12)$ if $c$ is odd, or $t-c \equiv 8$ or $11(\bmod 12)$ if $c$ is even. Put $m=(t-c+1) / 3$ and assume that $t$ is large enough to ensure that $m \geq 2 c-1$ (i.e. $t \geq 7 c-4)$. Then there exists an $(m, 3, c)$-system from which a Skolem-type sequence $T$ may be constructed that has length $2 t+1$, has zeros in the central $2 c-1$ positions and, for each $k \in\{c, c+1, \ldots, t\}$, the two positions occupied by $k$ are precisely $k$ apart. The sequence $T$ is used to replace the central $2 t+1$ zeros in each sequence $S$ of length $14 t+7$ given by Theorem 3.1. We denote the resulting sequence as $T \rightsquigarrow S$ ( $T$ into $S$ ), and this sequence has zeros in its central $2 c-1$ positions. These are then replaced by a sequence $Q$ of length $2 c-1$ to form $Q \rightsquigarrow(T \rightsquigarrow S)$, and a short sequence $R$ of further entries is appended at the right-hand end of this sequence to form a sequence $S^{\prime}=(Q \rightsquigarrow(T \rightsquigarrow S)) \wedge R$ (where $\wedge$ denotes appending). By choosing $c, Q$ and $R$ appropriately, it is possible to form hooked and split-hooked Skolem sequences $S^{\prime}$.

To illustrate the procedure, we explain how to convert a Skolem-type sequence $S$ of length 147 of the form described in Theorem 3.1, to a hooked Skolem sequence $S^{\prime}$ of order 74 . Note that for $k \in\{11,12, \ldots, 73\}$, the two positions in $S$ occupied by $k$ are precisely $k$ apart, and that $S$ has zeros in the central 21 positions. Next take a $(3,3,2)$-system (for example, the one previously described) and from it form a Skolem-type sequence $T$ of length 21 that has zeros in the central three positions and, for each $k \in\{2,3, \ldots, 10\}$, the two positions in $T$ occupied by $k$ are precisely $k$ apart. Replace the central 21 zeros of $S$ by the sequence $T$ to form $T \rightsquigarrow S$. Then $T \rightsquigarrow S$ has length 147, zeros in the central three positions and, for each $k \in\{2,3, \ldots, 73\}$, the two positions occupied by $k$ are precisely $k$ apart. Finally, replace the central three zeros in $T \rightsquigarrow S$ by the sequence $Q=(1,1,74)$, and append the sequence $R=(0,74)$ to the right-hand end of $Q \rightsquigarrow(T \rightsquigarrow S)$. The resulting sequence $S^{\prime}$ has length 149, has a zero in the penultimate position and, for each $k \in\{1,, 2, \ldots, 74\}$, the two positions occupied by $k$ are precisely $k$ apart. Hence $S^{\prime}$ is a 
hooked Skolem sequence of order 74 . Clearly different sequences $S$ give rise to different sequences $S^{\prime}$.

We now return to the general cases. For given $t$ it is obvious that different sequences $S$ will result in different sequences $S^{\prime}=(Q \rightsquigarrow(T \rightsquigarrow S)) \wedge R$. So, to extend the bound, it suffices to specify $c$ (and hence $T$ ), $Q$ and $R$, and to check the parity conditions for $t-c$. In the next two corollaries to Theorem 3.1, we establish the bound by tabulating appropriate $c$, $Q$ and $R$. We leave the reader to check the parity conditions for $t-c$ and that the sequence $S^{\prime}$ is of the required type.

Corollary 3.4. For sufficiently large $t \equiv 1$ or $2(\bmod 4)$, there are more than $(6.492)^{2 t+1}$ hooked Skolem sequences of order $7 t+4$.

Proof. Table 2 covers the possible values of $t$ modulo 12 .

Table 2: Construction of hooked Skolem sequences.

\begin{tabular}{c|c|c|c|c}
$t(\bmod 12)$ & $c$ & $Q$ & $R$ & $t \geq 7 c-4$ \\
\hline \hline 1,10 & 2 & $(1,1,7 t+4)$ & $(0,7 t+4)$ & $t \geq 10$ \\
2,5 & 3 & $(1,1,2,7 t+4,2)$ & $(0,7 t+4)$ & $t \geq 17$ \\
6,9 & 7 & $(2,4,2,5,6,4,3,7 t+4,5,3,6,1,1)$ & $(0,7 t+4)$ & $t \geq 45$
\end{tabular}

For each Skolem-type sequence $S$ of the form described in Theorem 3.1, the resulting sequence $S^{\prime}=(Q \rightsquigarrow(T \rightsquigarrow S)) \wedge R$ is a hooked Skolem sequence of order $7 t+4$.

Corollary 3.5. For sufficiently large $t \equiv 0$ or $3(\bmod 4)$, there are more than $(6.492)^{2 t+1}$ split-hooked Skolem sequences of order $7 t+5$.

Proof. Table 3 covers the possible values of $t$ modulo 12 .

Table 3: Construction of split-hooked Skolem sequences.

\begin{tabular}{c|c|c|c|c}
$t(\bmod 12)$ & $c$ & $Q$ & $R$ & $t \geq 7 c-4$ \\
\hline \hline 0,3 & 4 & $(1,1,7 t+5,3,7 t+4,0,3)$ & $(7 t+5,7 t+4,2,0,2)$ & $t \geq 24$ \\
4,7 & 5 & $(2,3,2,4,3,7 t+5,0$, & $(1,1,7 t+5,0,7 t+4)$ & $t \geq 31$ \\
& & $4,7 t+4)$ & & \\
8,11 & 9 & $(4,5,6,8,4,7,5,7 t+5,6$, & $(7 t+5,7 t+4,2,0,2)$ & $t \geq 59$ \\
& & $7 t+4,0,8,7,3,1,1,3)$ & &
\end{tabular}

For each Skolem-type sequence $S$ of the form described in Theorem 3.1, the resulting sequence $S^{\prime}=(Q \rightsquigarrow(T \rightsquigarrow S)) \wedge R$ is a split-hooked Skolem sequence of order $7 t+5$.

The bound obtained in each of the preceding four corollaries only applies to restricted parts of the appropriate residue classes. We believe that it is possible to extend the bound to all possible residue classes in each case. We do not give a proof of this because our argument breaks into a considerable number of subcases. However, to support our contention, we give one example for split Skolem sequences. Corollary 3.2 gives the bound 
$(6.492)^{2 t+1}$ when $n=7 t+3$ and $t \equiv 0$ or $3(\bmod 4)$, thereby dealing with $n \equiv 3$ or 24 $(\bmod 28)$. The necessary and sufficient conditions on $n$ for the existence of a split Skolem sequence of order $n$ may be written as $n \equiv 0,3,4,7,8,11,12,15,16,19,20,23,24$ or 27 $(\bmod 28)$. For our example, we show how the bound may be extended to $n \equiv 0$ or 7 $(\bmod 28)$.

Put $n=7 t+7$ where $t \equiv 0$ or $3(\bmod 4)$. Take $S$ to be a Skolem-type sequence as described in Theorem 3.1. Depending on the residue of $t$ modulo 12, take $c$ as in Table 4 and put $m=(t-c+1) / 3$. For $t \geq 7 c-4$, use an $(m, 3, c)$-system to construct a Skolemtype sequence $T$ of length $2 t+1$ having zeros in the central $2 c-1$ positions and such that for each $k \in\{c, c+1, \ldots, t\}$, the two positions occupied by $k$ are precisely $k$ apart. Take $Q$ and $R$ as specified in the table and form $S^{\prime}=(Q \rightsquigarrow(T \rightsquigarrow S)) \wedge R$. Then $S^{\prime}$ is a split Skolem sequence of length $14 t+15$ (i.e. of order $n=7 t+7$ ). Hence, for all sufficiently large $t$, there are more than $(6.492)^{2 t+1}$ split Skolem sequences of order $n=7 t+7$ where $t \equiv 0$ or $3(\bmod 4)$, so that $n \equiv 0$ or $7(\bmod 28)$.

Table 4: Further split Skolem sequences.

\begin{tabular}{c|c|c|c|c}
$t(\bmod 12)$ & $c$ & $Q$ & $R$ & $t \geq 7 c-4$ \\
\hline \hline \multirow{2}{*}{8,7} & \multirow{2}{*}{5} & $(4,7 t+7,3,7 t+6,4$, & $(7 t+7,7 t+6,7 t+4$, & $t \geq 31$ \\
& & $3,7 t+4,7 t+5,0)$ & $2,7 t+5,2,1,1)$ & \\
& \multirow{3}{*}{0} & $(1,1,3,8,4,3,7 t+7$, & $(5,7 t+7,2,7 t+6,2$, & $t \geq 59$ \\
& & $7,4,7 t+6,6,8,0$, & $5,7 t+5,7 t+4)$ & \\
& \multirow{4}{*}{16} & $7 t+5,7,7 t+4,6)$ & & $t \geq 108$ \\
& & $(11,8,4,7,13,9,4,14,10$, & $(5,7 t+7,2,7 t+6,2$, & $t$ \\
& & $8,7,11,12,7 t+7,9,15$, & $5,7 t+5,7 t+4)$ & \\
& & $7 t+6,13,10,0,7 t+5,14$, & &
\end{tabular}

There are methods other than the one described above for generating Skolem-type sequences from additive permutations. For certain orders the construction technique given below can give improved bounds.

Suppose that $s>\ell>0$ and that $\mathcal{S}$ is a Skolem-type sequence of length $2 s+1$ having zeros in the central $2 \ell-1$ positions, and such that for $k \in\{\ell, \ell+1, \ldots, s\}$ the two positions where $k$ appears in $\mathcal{S}$ are precisely $k$ apart. If $\ell=1$ then $\mathcal{S}$ is a split Skolem sequence of order $s$ (and such a sequence exists if $s \equiv 0$ or $3(\bmod 4))$, otherwise the earlier discussion following Corollary 3.3 shows that such a sequence exists when $s-\ell \equiv 2$ or $11(\bmod 12)$ if $\ell$ is odd, or $s-\ell \equiv 8$ or $11(\bmod 12)$ if $\ell$ is even, provided that $s \geq 7 \ell-4$. Let $\mathcal{S}$ be indexed by $[-s, s]$.

\section{Construction 3.6.}

- For $j=\ell, \ell+1, \ldots, s$, denote by $a_{j}, b_{j}$ (with $a_{j}<b_{j}$ ) the positions in $\mathcal{S}$ occupied by the entry $j$, so that $b_{j}-a_{j}=j$.

- For each $j=\ell, \ell+1, \ldots, s$, let $\pi_{j}$ be an additive permutation on $[-t, t]$, and denote by $\Pi$ the ordered $(s-\ell+1)$-tuple $\left(\pi_{\ell}, \pi_{\ell+1}, \ldots, \pi_{s}\right)$. 
- Form a new sequence $\mathcal{S}_{\Pi}$ indexed by $[-((2 t+1) s+t),(2 t+1) s+t]$ by placing the entry $(2 t+1) j+\pi_{j}(i)$ at positions $(2 t+1) a_{j}+i$ and $(2 t+1) b_{j}+i+\pi_{j}(i)$ for $j=\ell, \ell+1, \ldots, s$ and $i \in[-t, t]$. For each $j$ these entries cover the interval $[(2 t+1) j-t,(2 t+1) j+t]$, and so they collectively cover $[(2 t+1) \ell-t,(2 t+1) s+t]$. These entries cover the positions

$$
[-((2 t+1) s+t),(2 t+1) s+t] \backslash[-(2 t+1) \ell+t+1,(2 t+1) \ell-t-1] .
$$

Place zeros in the vacant positions. Then $\mathcal{S}_{\Pi}$ is a Skolem-type sequence of length $2(2 s t+s+t)+1$ having zeros in the central $2((2 t+1) \ell-t)-1$ positions, and such that for $k \in[(2 t+1) \ell-t,(2 t+1) s+t]$ the two positions where $k$ appears in $\mathcal{S}_{\Pi}$ are precisely $k$ apart.

- Now suppose that $t \equiv 0$ or $3(\bmod 4)$ and let $\mathcal{P}$ be a split Skolem sequence of order $t$, indexed by $[-t, t]$. Apply the previous three steps to $\mathcal{P}$ using additive permutations $\sigma_{j}$ on $[-(\ell-1), \ell-1]$ for $j=1,2, \ldots, t$ to form a new sequence $\mathcal{P}_{\Sigma}$, where $\Sigma$ is the ordered t-tuple $\left(\sigma_{1}, \sigma_{2}, \ldots, \sigma_{t}\right)$. Then $\mathcal{P}_{\Sigma}$ is a Skolem-type sequence of length $2(2 t(\ell-1)+t+(\ell-1))+1=2((2 t+1) \ell-t)-1$ having zeros in the central $2((2(\ell-1)+1)-(\ell-1)))-1=2 \ell-1$ positions, and such that for $k \in[\ell,(2 t+1) \ell-t-1]$ the two positions where $k$ appears in $\mathcal{P}_{\Sigma}$ are precisely $k$ apart. Replace the central zeros of $\mathcal{S}_{\Pi}$ by $\mathcal{P}_{\Sigma}$ to form the sequence $\mathcal{P}_{\Sigma} \rightsquigarrow \mathcal{S}_{\Pi}$.

Then $\mathcal{P}_{\Sigma} \rightsquigarrow \mathcal{S}_{\Pi}$ is a Skolem-type sequence of length $2(2 s t+s+t)+1$ having zeros in the central $2 \ell-1$ positions, and such that for $k \in\{\ell, \ell+1, \ldots, 2 s t+s+t\}$ the two positions where $k$ appears in $\mathcal{P}_{\Sigma} \rightsquigarrow \mathcal{S}_{\Pi}$ are precisely $k$ apart.

Given a sequence $\mathcal{P}_{\Sigma} \rightsquigarrow \mathcal{S}_{\Pi}$ constructed in this fashion for given $\ell, s$ and $t$, the ingredients $\mathcal{S}, \Pi, \mathcal{P}$ and $\Sigma$ may be recovered by considering entries and positions. Suppose that entry $e>0$ occupies positions $a$ and $b$ with $a<b$. If $e \geq(2 t+1) \ell-t$ then $e=(2 t+1) j+\pi_{j}(i)$ for some $i$ and $j$, and $j=\lfloor(e+t) /(2 t+1)\rfloor$, while $a=(2 t+1) a_{j}+i$, so $a_{j}=\lfloor(a+t) /(2 t+1)\rfloor$. Similarly, $b_{j}=\lfloor(b+t) /(2 t+1)\rfloor$, while $i, \pi_{j}(i) \in[-t, t]$ are given by $i \equiv a(\bmod 2 t+1)$ and $\pi_{j}(i) \equiv e(\bmod 2 t+1)$. This process recovers $\mathcal{S}$ and $\Pi$. If $e \leq(2 t+1) \ell-t-1=(2(\ell-1)+1) t+(\ell-1)$, then $\mathcal{P}$ and $\Sigma$ may be recovered in the same way from $\lfloor(e+(\ell-1)) /(2 \ell-1)\rfloor$, etc.

Hence, varying any of $\mathcal{S}, \Pi, \mathcal{P}$ and $\Sigma$ will yield different Skolem-type sequences $\mathcal{P}_{\Sigma} \rightsquigarrow$ $\mathcal{S}_{\Pi}$. Disregarding variation due to selection of $\mathcal{S}, \mathcal{P}$, and $\Sigma$, the following result is obtained.

Theorem 3.7. Suppose that there exists a Skolem-type sequence $\mathcal{S}$ of length $2 s+1$ having zeros in the central $2 \ell-1$ positions, and such that for $k \in\{\ell, \ell+1, \ldots, s\}$ the two positions where $k$ appears in $\mathcal{S}$ are precisely $k$ apart. Then for $t \equiv 0$ or $3(\bmod 4)$ there are at least $\left(z_{2 t+1}\right)^{s-\ell+1}$ Skolem-type sequences $\mathcal{P}_{\Sigma} \rightsquigarrow \mathcal{S}_{\Pi}$ of length $2(2 s t+s+t)+1$ having zeros in the central $2 \ell-1$ positions, and such that for $k \in\{\ell, \ell+1, \ldots, 2 s t+s+t\}$ the two positions where $k$ appears in $\mathcal{P}_{\Sigma} \rightsquigarrow \mathcal{S}_{\Pi}$ are precisely $k$ apart.

This result generates lower bounds for the numbers of pure, split, hooked and splithooked Skolem sequences of various orders. Recall from Corollary 2.2 that for sufficiently large $t, z_{2 t+1}>(3.246)^{2 t+1}$.

Corollary 3.8. If $s \equiv 0$ or $3(\bmod 4)$, then for all sufficiently large $t \equiv 0$ or $3(\bmod 4)$, the number of split Skolem sequences of order $2 s t+s+t$ is greater than $(3.246)^{2 s t+s}$. 
Proof. Invoke the above construction with $\ell=1$.

As an example, taking $s=3$ gives that for all sufficiently large $t \equiv 0$ or $3(\bmod 4)$, the number of split Skolem sequences of order $n=7 t+3$ is greater than $(3.246)^{6 t+3}$. This is substantially better than the bound given earlier in Corollary 3.2, although this approach does not appear to offer the generalization to $n \equiv 0$ or $3(\bmod 4)$ mentioned in connection with the previous method.

If the central zero in a split Skolem sequence of order $n$ is replaced by $n+1$ and an additional entry $n+1$ is placed at either the start or the end of the sequence, then a pure Skolem sequence of order $n+1$ is formed. Applying this adaptation to the split Skolem sequence constructed in Corollary 3.8 gives the following result.

Corollary 3.9. If $s \equiv 0$ or $3(\bmod 4)$, then for all sufficiently large $t \equiv 0$ or $3(\bmod 4)$, the number of pure Skolem sequences of order $2 s t+s+t+1$ is greater than $(3.246)^{2 s t+s}$.

To deal with hooked and split-hooked Skolem sequences, we may replace the central $2 \ell-1$ zeros of $\mathcal{P}_{\Sigma} \rightsquigarrow \mathcal{S}_{\Pi}$ with an appropriate sequence $Q$, and append a short sequence $R$ to the right hand end to form $\left(Q \rightsquigarrow\left(\mathcal{P}_{\Sigma} \rightsquigarrow \mathcal{S}_{\Pi}\right)\right) \wedge R$.

Corollary 3.10. If $s \equiv 1$ or $2(\bmod 4)$ and $s \geq 45$, then for all sufficiently large $t \equiv$ 0 or $3(\bmod 4)$, the number of hooked Skolem sequences of order $2 s t+s+t+1$ is greater than $(3.246)^{(s-6)(2 t+1)}$.

Proof. Given $s$ and $t$, put $m=2 s t+s+t$. The sequences $Q$ and $R$ for possible values of $s$ modulo 12 are covered by using Table 2 with $t$ replaced by $s, c$ replaced by $\ell$ and $7 t+3$ replaced by $m$. Note that in Table $2, c \leq 7$ and so we may assume that $\ell \leq 7$. In each case, the resulting sequence $\left(Q \rightsquigarrow\left(\mathcal{P}_{\Sigma} \rightsquigarrow \mathcal{S}_{\Pi}\right)\right) \wedge R$ is a hooked Skolem sequence of order $m+1=2 s t+s+t+1$, and the result follows.

Corollary 3.11. If $s \equiv 0$ or $3(\bmod 4)$ and $s \geq 59$, then for all sufficiently large $t \equiv$ 0 or $3(\bmod 4)$, the number of split-hooked Skolem sequences of order $2 s t+s+t+2$ is greater than $(3.246)^{(s-8)(2 t+1)}$.

Proof. Given $s$ and $t$, put $m=2 s t+s+t$. The sequences $Q$ and $R$ for possible values of $s$ modulo 12 are covered by using Table 3 with $t$ replaced by $s, c$ replaced by $\ell$ and $7 t+3$ replaced by $m$. Note that in Table $3, c \leq 9$ and so we may assume that $\ell \leq 9$. In each case, the resulting sequence $\left(Q \rightsquigarrow\left(\mathcal{P}_{\Sigma} \rightsquigarrow \mathcal{S}_{\Pi}\right)\right) \wedge R$ is a split-hooked Skolem sequence of order $m+2=2 s t+s+t+2$, and the result follows.

\section{Completing the proof of Theorem 2.1}

As previously described, the arithmetic is simplified by subtracting $t-s-a$ from all the entries in $R$ and $S$ to obtain equivalent arrays $R^{\prime}$ and $S^{\prime}$, where $R^{\prime}$ has entries ranging from $-(s+2 a)$ to $s+2 a$, and $S^{\prime}$ has entries ranging from $-(s-1)$ to $s-1$. Transversals are sought in these arrays that cover the entry values from $-(s+a)$ to $s+a$ inclusive. We renumber the rows and columns so that for $R^{\prime}$ the row and column numbers run from 0 to $r-1=s+2 a$ and for $S^{\prime}$ they run from 0 to $s-1$. The entry in cell $(i, j)$ of $R^{\prime}$ is then $i+j-(s+2 a)$, and that in cell $(i, j)$ of $S^{\prime}$ is $i+j-(s-1)$. The identification of transversals falls into several cases. 
Table 5: Case 1 ( $r$ even), transversal in $S^{\prime}$.

\begin{tabular}{l|l|l|l|l} 
& Range & Row & Column & Entry \\
\hline \hline (a) & $j=0, \ldots, \frac{s-1}{2}$ & $j$ & $\frac{s-1}{2}+j$ & $-\frac{s-1}{2}+2 j$ \\
\hline (b) $\dagger$ & $j=0, \ldots, \frac{s-1}{2}-1$ & $\frac{s-1}{2}+1+j$ & $j$ & $-\frac{s-1}{2}+1+2 j$
\end{tabular}

Case 1: $r$ even. Since $r$ is even, $s$ must be odd. Table 5 identifies a suitable transversal in $S^{\prime}$. Line (b) of the table, marked with $\dagger$, is omitted when $s=1$. In $S^{\prime}$, and subject to $\dagger$, line (a) of the table covers rows 0 to $\frac{s-1}{2}$, and line (b) covers rows $\frac{s-1}{2}+1$ to $s-1$. For columns, line (b) covers 0 to $\frac{s-1}{2}-1$, and line (a) covers $\frac{s-1}{2}$ to $s-1$. As regards entries, lines (a) and (b) together cover $-\frac{s-1}{2}$ to $\frac{s-1}{2}$.

Subcase 1.1: $\boldsymbol{r} \equiv \mathbf{0}(\bmod 4)$. Table 6 identifies a suitable transversal in $R^{\prime}$. The line of the table marked with $*$ is omitted when $s=b-2$, and the line marked $\dagger$ is omitted when $s=1$. Subject to $*$ and $\dagger$, rows, columns and entries of $R^{\prime}$ are covered by lines of the

Table 6: Subcase $1.1(r \equiv 0(\bmod 4))$, transversal in $R^{\prime}$.

\begin{tabular}{l|l|l|l|l} 
& Range & Row & Column & Entry \\
\hline \hline (a) & $j=0, \ldots, \frac{r}{4}-1$ & $j$ & $a+j$ & $-a-s+2 j$ \\
\hline (b) & $j=0, \ldots, a-\frac{r}{4}$ & $\frac{r}{4}+j$ & $a+\frac{r}{4}+s+j$ & $\frac{s-1}{2}+1+2 j$ \\
\hline (c) & $j=0, \ldots, \frac{r}{4}-1$ & $a+1+j$ & $j$ & $-a-s+1+2 j$ \\
\hline (d) & $j=0, \ldots, \frac{s-1}{2}$ & $a+\frac{r}{4}+1+j$ & $a+\frac{r}{4}+\frac{s-1}{2}+j$ & $a+1+2 j$ \\
\hline (e) $\dagger$ & $j=0, \ldots, \frac{s-1}{2}-1$ & $a+\frac{r}{4}+\frac{s-1}{2}+$ & $a+\frac{r}{4}+j$ & $a+2+2 j$ \\
\hline (f) $*$ & $j=0, \ldots, a-\frac{r}{4}-1$ & $a+\frac{r}{4}+s+$ & $\frac{r}{4}+j$ & $\frac{s-1}{2}+2+2 j$ \\
& & $1+j$ & &
\end{tabular}

table in the following orders, where notation such as (a\&c) means that entries from lines (a) and (c) are interleaved and taken together. Rows 0 to $2 a+s$ by lines (a)(b)(c)(d)(e)(f) in that order. Columns 0 to $2 a+s$ by lines (c)(f)(a)(e)(d)(b) in that order. Entries $-a-s$ to $-\frac{s-1}{2}-1$ by lines (a\&c), and entries $\frac{s-1}{2}+1$ to $a+s$ by lines (b\&f)(d\&e) in that order; the remaining entries required to complete the transversal values from $-(s+a)$ to $(s+a)$ come from the transversal in $S^{\prime}$.

Subcase 1.2: $r \equiv 2(\bmod 4)$. Table 7 identifies a suitable transversal in $R^{\prime}$. The line of the table marked with $\dagger$ is omitted when $s=1$. Subject to $\dagger$, rows, columns and entries of $R^{\prime}$ are covered by lines of the table in the following orders. Rows 0 to $2 a+s$ by lines $(\mathrm{a})(\mathrm{b})(\mathrm{c})(\mathrm{d})(\mathrm{e})(\mathrm{f})$ in that order. Columns 0 to $2 a+s$ by lines $(\mathrm{c})(\mathrm{f})(\mathrm{a})(\mathrm{e})(\mathrm{d})(\mathrm{b})$ in that order. Entries $-a-s$ to $-\frac{s-1}{2}-1$ by lines ( $\& \mathrm{c}$ ), and entries $\frac{s-1}{2}+1$ to $a+s$ by lines (b\&f)(d\&e) in that order; the remaining entries required to complete the transversal values from $-(s+a)$ to $(s+a)$ come from the transversal in $S^{\prime}$.

Case 2: $r$ odd. If $r$ is odd then $s$ must be even. If $s=0$ then $r=b$ and $R$ is a copy of $A_{b}$ 
Table 7: Subcase $1.2(r \equiv 2(\bmod 4))$, transversal in $R^{\prime}$.

\begin{tabular}{l|l|l|l|l} 
& Range & Row & Column & Entry \\
\hline \hline (a) & $j=0, \ldots, \frac{r+2}{4}-1$ & $j$ & $a+j$ & $-a-s+2 j$ \\
\hline (b) & $j=0, \ldots, a-\frac{r+2}{4}$ & $\frac{r+2}{4}+j$ & $\begin{array}{r}a+\frac{r+2}{4}+ \\
s+j\end{array}$ & $\frac{s-1}{2}+2+2 j$ \\
\hline (c) & $j=0, \ldots, \frac{r+2}{4}-2$ & $a+1+j$ & $j$ & $-a-s+1+2 j$ \\
\hline (d) & $j=0, \ldots, \frac{s-1}{2}$ & $a+\frac{r+2}{4}+j$ & $a+\frac{r+2}{4}+$ & $a+1+2 j$ \\
\hline (e) $\dagger$ & $j=0, \ldots, \frac{s-1}{2}-1$ & $a+\frac{r+2}{4}+\frac{s-1}{2}+$ & $a+\frac{r+2}{4}+j$ & $a+2+2 j$ \\
\hline (f) & $j=0, \ldots, a-\frac{r+2}{4}$ & $a+\frac{r+2}{4}+s+j$ & $\frac{r+2}{4}-1+j$ & $\frac{s-1}{2}+1+2 j$
\end{tabular}

(with rows and columns appropriately renumbered) and any one of the transversals already identified in $A_{b}$ provides a suitable transversal in $R$. So throughout Case 2, we may assume that $s>0$, and then Table 8 identifies a suitable transversal in $S^{\prime}$. Line (b) of the table, marked with $\dagger$, is omitted when $s=2$. Subject to $\dagger$, rows, columns and entries of $S^{\prime}$ are

Table 8: Case 2 ( $r$ odd), transversal in $S^{\prime}$.

\begin{tabular}{l|l|l|l|l} 
& Range & Row & Column & Entry \\
\hline \hline (a) & $j=0, \ldots, \frac{s}{2}-1$ & $j$ & $\frac{s}{2}-1+j$ & $-\frac{s}{2}+2 j$ \\
\hline (b) $\dagger$ & $j=0, \ldots, \frac{s}{2}-2$ & $\frac{s}{2}+j$ & $j$ & $-\frac{s}{2}+1+2 j$ \\
\hline (c) & single cell & $s-1$ & $s-1$ & $s-1$
\end{tabular}

covered by lines of the table in the following orders. Rows 0 to $s-1$ by lines (a)(b)(c) in that order. Columns 0 to $s-1$ by lines (b)(a)(c) in that order. Entries $-\frac{s}{2}$ to $\frac{s}{2}-2$ by lines (a\&b), and entry $s-1$ by line (c).

To deal with $R^{\prime}$, we consider four subcases depending on the values of $r$ and $s$ modulo 4 .

Subcase 2.1: $\boldsymbol{r} \equiv \mathbf{1}, \boldsymbol{s} \equiv \mathbf{0}(\bmod 4)$. These conditions imply that $b \equiv 1(\bmod 4)$ and we may assume that $s \geq 4$. Table 9 identifies a suitable transversal in $R^{\prime}$. Lines of the table marked with $*$ are omitted when $s=b-1$, and lines marked with $\dagger$ are omitted when $s=4$. Subject to $*$ and $\dagger$, rows, columns and entries of $R^{\prime}$ are covered by lines of the table in the following orders. Rows 0 to $2 a+s$ by lines $(\mathrm{a})(\mathrm{b})(\mathrm{c})(\mathrm{d})(\mathrm{e})(\mathrm{f})(\mathrm{g})(\mathrm{h})(\mathrm{i})(\mathrm{j})(\mathrm{k})(\mathrm{l})(\mathrm{m})$ in that order. Columns 0 to $2 a+s$ by lines $(k)(f)(i)(g)(e)(b)(d)(1)(c)(m)(a)(h)(j)$ in that order. Entries $-a-s$ to $-\frac{s}{2}-1$ by lines $(\mathrm{b} \& \mathrm{f})(\mathrm{e})(\mathrm{d} \& \mathrm{~g})(\mathrm{i})(\mathrm{c})(\mathrm{a} \& \mathrm{k})$ in that order, entries $\frac{s}{2}-1$ to $s-2$ by lines (h\&l), and entries $s$ to $a+s$ by lines (j\&m); the remaining entries required to complete the transversal values from $-(s+a)$ to $(s+a)$ come from the transversal in $S^{\prime}$.

Subcase 2.2: $\boldsymbol{r} \equiv \mathbf{1}, \boldsymbol{s} \equiv \mathbf{2}(\bmod 4)$. These conditions imply that $b \equiv 3(\bmod 4)$ and $a$ is odd. Table 10 identifies a suitable transversal in $R^{\prime}$ when $s \geq 6$. The line of the table 
Table 9: Subcase $2.1(r \equiv 1, s \equiv 0(\bmod 4))$, transversal in $R^{\prime}$.

\begin{tabular}{l|l|l|l|l} 
& Range & Row & Column & Entry \\
\hline \hline (a) $*$ & $j=0, \ldots, \frac{a}{2}-\frac{s}{4}-1$ & $j$ & $a+s+j$ & $-a+2 j$ \\
\hline (b) & $j=0, \ldots, \frac{s}{4}-1$ & $\frac{a}{2}-\frac{s}{4}+j$ & $\frac{a}{2}+\frac{s}{4}+j$ & $-a-s+2 j$ \\
\hline (c) & single cell & $\frac{a}{2}$ & $\frac{a}{2}+s-1$ & $-a-1$ \\
\hline (d) $\dagger$ & $j=0, \ldots, \frac{s}{4}-2$ & $\frac{a}{2}+1+j$ & $\frac{a}{2}+\frac{s}{2}+j$ & $-a-\frac{s}{2}+1+2 j$ \\
\hline (e) & single cell & $\frac{a}{2}+\frac{s}{4}$ & $\frac{a}{2}+\frac{s}{4}-1$ & $-a-\frac{s}{2}-1$ \\
\hline (f) $\dagger$ & $j=0, \ldots, \frac{s}{4}-2$ & $\frac{a}{2}+\frac{s}{4}+1+j$ & $\frac{a}{2}-\frac{s}{4}+j$ & $-a-s+1+2 j$ \\
\hline (g) $\dagger$ & $j=0, \ldots, \frac{s}{4}-2$ & $\frac{a}{2}+\frac{s}{2}+j$ & $\frac{a}{2}+j$ & $-a-\frac{s}{2}+2 j$ \\
\hline (h) & $j=0, \ldots, \frac{s}{4}-1$ & $\frac{a}{2}+\frac{3 s}{4}-1+j$ & $\frac{3 a}{2}+\frac{3 s}{4}+j$ & $\frac{s}{2}-1+2 j$ \\
\hline (i) & single cell & $\frac{a}{2}+s-1$ & $\frac{a}{2}-1$ & $-a-2$ \\
\hline (j) & $j=0, \ldots, \frac{a}{2}$ & $\frac{a}{2}+s+j$ & $\frac{3 a}{2}+s+j$ & $s+2 j$ \\
\hline (k) $*$ & $j=0, \ldots, \frac{a}{2}-\frac{s}{4}-1$ & $a+s+1+j$ & $j$ & $-a+1+2 j$ \\
\hline (l) & $j=0, \ldots, \frac{s}{4}-1$ & $\frac{3 a}{2}+\frac{3 s}{4}+1+j$ & $\frac{a}{2}+\frac{3 s}{4}-1+j$ & $\frac{s}{2}+2 j$ \\
\hline (m) & $j=0, \ldots, \frac{a}{2}-1$ & $\frac{3 a}{2}+s+1+j$ & $\frac{a}{2}+s+j$ & $s+1+2 j$ \\
\hline
\end{tabular}

marked with $*$ is omitted when $s=b-1$, and the line of the table marked with $\dagger$ is omitted when $s=6$. Subject to $*$ and $\dagger$, rows, columns and entries of $R^{\prime}$ are covered by lines of the table in the following orders. Rows 0 to $2 a+s$ by lines $(\mathrm{a})(\mathrm{b})(\mathrm{c})(\mathrm{d})(\mathrm{e})(\mathrm{f})(\mathrm{g})(\mathrm{h})(\mathrm{i})(\mathrm{j})(\mathrm{k})(\mathrm{l})(\mathrm{m})$ in that order. Columns 0 to $2 a+s$ by lines $(\mathrm{k})(\mathrm{f})(\mathrm{i})(\mathrm{g})(\mathrm{e})(\mathrm{b})(\mathrm{d})(\mathrm{l})(\mathrm{c})(\mathrm{m})(\mathrm{a})(\mathrm{h})(\mathrm{j})$ in that order. Entries $-a-s$ to $-\frac{s}{2}-1$ by lines $(\mathrm{b} \& \mathrm{f})(\mathrm{e})(\mathrm{d} \& \mathrm{~g})(\mathrm{c})(\mathrm{i})(\mathrm{a} \& \mathrm{k})$ in that order, entries $\frac{s}{2}-1$ to $s-2$ by lines (h\&l), and entries $s$ to $a+s$ by lines (j\&m); the remaining entries required to complete the transversal values from $-(s+a)$ to $(s+a)$ come from the transversal in $S^{\prime}$.

The case $s=2$ may be obtained from the table by omitting lines (b), (d), (e), (f), (g) and (1). Subject to $*$ (i.e. when $b=3$ ), rows, columns and entries of $R^{\prime}$ are covered by lines of the table in the following orders. Rows 0 to $2 a+2$ by lines $(\mathrm{a})(\mathrm{c})(\mathrm{h})(\mathrm{i})(\mathrm{j})(\mathrm{k})(\mathrm{m})$ in that order. Columns 0 to $2 a+2$ by lines $(\mathrm{k})(\mathrm{i})(\mathrm{c})(\mathrm{m})(\mathrm{a})(\mathrm{h})(\mathrm{j})$ in that order. Entries $-a-2$ to -2 by lines (c)(i)(a\&k) in that order, entry 0 by line (h), and entries 2 to $a+2$ by lines $(\mathrm{j} \& \mathrm{~m})$; the remaining entries required to complete the transversal values from $-(a+2)$ to $(a+2)$ come from the transversal in $S^{\prime}$.

Subcase 2.3: $\boldsymbol{r} \equiv \mathbf{3}, \boldsymbol{s} \equiv \mathbf{0}(\bmod 4)$. These conditions imply that $b \equiv 3(\bmod 4)$ and $a$ is odd. Table 11 identifies a suitable transversal in $R^{\prime}$. The line of the table marked with $*$ is omitted when $s=b-3$, and the lines of the table marked with $\dagger$ are omitted when $s=4$. Subject to $*$ and $\dagger$, rows, columns and entries of $R^{\prime}$ are covered by lines of the table in the following orders. Rows 0 to $2 a+s$ by lines $(\mathrm{a})(\mathrm{b})(\mathrm{c})(\mathrm{d})(\mathrm{e})(\mathrm{f})(\mathrm{g})(\mathrm{h})(\mathrm{i})(\mathrm{j})(\mathrm{k})(\mathrm{l})(\mathrm{m})$ in that order. Columns 0 to $2 a+s$ by lines $(\mathrm{k})(\mathrm{f})(\mathrm{i})(\mathrm{g})(\mathrm{e})(\mathrm{b})(\mathrm{d})(\mathrm{l})(\mathrm{c})(\mathrm{m})(\mathrm{a})(\mathrm{h})(\mathrm{j})$ in that order. Entries $-a-s$ to $-\frac{s}{2}-1$ by lines $(\mathrm{b} \& \mathrm{f})(\mathrm{e})(\mathrm{d} \& \mathrm{~g})(\mathrm{i})(\mathrm{c})(\mathrm{a} \& \mathrm{k})$ in that order, entries $\frac{s}{2}-1$ to $s-2$ by lines (h\&l), and entries $s$ to $a+s$ by lines (j\&m); the remaining entries required to complete the transversal values from $-(s+a)$ to $(s+a)$ come from the transversal in $S^{\prime}$. 
Table 10: Subcase $2.2(r \equiv 1, s \equiv 2(\bmod 4))$, transversal in $R^{\prime}$.

\begin{tabular}{l|l|l|l|l} 
& Range & Row & Column & Entry \\
\hline \hline (a) $*$ & $j=0, \ldots, \frac{a-1}{2}-$ & $j$ & $a+s+j$ & $-a+2 j$ \\
\hline (b) & $j=0, \ldots, \frac{s-2}{4}-1$ & $\frac{a-1}{2}-\frac{s-2}{4}+j$ & $\frac{a-1}{2}+\frac{s-2}{4}+$ & $-a-s+2 j$ \\
\hline (c) & single cell & $\frac{a-1}{2}$ & \\
\hline (d) & $j=0, \ldots, \frac{s-2}{4}-1$ & $\frac{a-1}{2}+1+j$ & $\frac{a-1}{2}+\frac{s}{2}+j$ & $-a-\frac{s}{2}+2 j$ \\
\hline (e) & single cell & $\frac{a-1}{2}+\frac{s-2}{4}+1$ & $\frac{a-1}{2}+\frac{s-2}{4}$ & $-a-\frac{s}{2}-1$ \\
\hline (f) & $j=0, \ldots, \frac{s-2}{4}-1$ & $\frac{a-1}{2}+\frac{s-2}{4}+$ & $\frac{a-1}{2}-\frac{s-2}{4}+j$ & $-a-s+1+2 j$ \\
\hline (g) $\dagger$ & $j=0, \ldots, \frac{s-2}{4}-2$ & $\frac{a-1}{2}+\frac{s}{2}+1+j$ & $\frac{a-1}{2}+1+j$ & $-a-\frac{s}{2}+1+2 j$ \\
\hline (h) & $j=0, \ldots, \frac{s-2}{4}$ & $\frac{a-1}{2}+\frac{3 s-2}{4}+j$ & $\frac{3 a+1}{2}+\frac{3 s-2}{4}+j$ & $\frac{s}{2}-1+2 j$ \\
\hline (i) & single cell & $\frac{a-1}{2}+s$ & $\frac{a-1}{2}$ & $-a-1$ \\
\hline (j) & $j=0, \ldots, \frac{a-1}{2}$ & $\frac{a-1}{2}+s+1+j$ & $\frac{3 a+1}{2}+s+j$ & $s+1+2 j$ \\
\hline (k) $*$ & $j=0, \ldots, \frac{a-1}{2}-$ & $a+s+1+j$ & $j$ & $-a+1+2 j$ \\
\hline (l) & $j=0, \ldots, \frac{s-2}{4}-1$ & $\frac{3 a+1}{2}+\frac{3 s-2}{4}+$ & $\frac{a-1}{2}+\frac{3 s-2}{4}+j$ & $\frac{s}{2}+2 j$ \\
\hline (m) & $j=0, \ldots, \frac{a-1}{2}$ & $\frac{3 a+1}{2}+s+j$ & $\frac{a-1}{2}+s+j$ & $s+2 j$ \\
\hline
\end{tabular}

Subcase 2.4: $\boldsymbol{r} \equiv \mathbf{3}, \boldsymbol{s} \equiv \mathbf{2}(\bmod 4)$. These conditions imply that $b \equiv 1(\bmod 4)$ and $a$ is even. Table 12 identifies a suitable transversal in $R^{\prime}$ when $s \geq 6$. The line of the table marked with $*$ is omitted when $s=b-3$, and the line of the table marked with $\dagger$ is omitted when $s=6$. Subject to $*$ and $\dagger$, rows, columns and entries of $R^{\prime}$ are covered by lines of the table in the following orders. Rows 0 to $2 a+s$ by lines $(\mathrm{a})(\mathrm{b})(\mathrm{c})(\mathrm{d})(\mathrm{e})(\mathrm{f})(\mathrm{g})(\mathrm{h})(\mathrm{i})(\mathrm{j})(\mathrm{k})(\mathrm{l})(\mathrm{m})$ in that order. Columns 0 to $2 a+s$ by lines $(k)(f)(i)(g)(e)(b)(d)(l)(c)(m)(a)(h)(j)$ in that order. Entries $-a-s$ to $-\frac{s}{2}-1$ by lines $(\mathrm{b} \& \mathrm{f})(\mathrm{e})(\mathrm{d} \& \mathrm{~g})(\mathrm{i})(\mathrm{c})(\mathrm{a} \& \mathrm{k})$ in that order, entries $\frac{s}{2}-1$ to $s-2$ by lines (h\&l), and entries $s$ to $a+s$ by lines ( $\mathrm{j} \& \mathrm{~m})$; the remaining entries required to complete the transversal values from $-(s+a)$ to $(s+a)$ come from the transversal in $S^{\prime}$.

The case $s=2$ may be obtained from the table by omitting lines (b), (d), (e), (f), (g) and (h). Subject to $*$ (i.e. when $b=5$ ), rows, columns and entries of $R^{\prime}$ are covered by lines of the table in the following orders. Rows 0 to $2 a+2$ by lines (a)(c)(i)(j)(k)(l)(m) in that order. Columns 0 to $2 a+2$ by lines $(\mathrm{k})(\mathrm{i})(\mathrm{l})(\mathrm{c})(\mathrm{m})(\mathrm{a})(\mathrm{j})$ in that order. Entries $-a-2$ to -2 by lines (i)(c)(a\&k) in that order, entry 0 by line (1), and entries 2 to $a+2$ by lines (j\&m); the remaining entries required to complete the transversal values from $-(a+2)$ to $(a+2)$ come from the transversal in $S^{\prime}$.

This concludes the proof of Theorem 2.1. 
Table 11: Subcase $2.3(r \equiv 3, s \equiv 0(\bmod 4))$, transversal in $R^{\prime}$.

\begin{tabular}{l|l|l|l|l} 
& Range & Row & Column & Entry \\
\hline \hline (a) & $j=0, \ldots, \frac{a-1}{2}-\frac{s}{4}$ & $j$ & $a+s+j$ & $-a+2 j$ \\
\hline (b) & $j=0, \ldots, \frac{s}{4}-1$ & $\frac{a+1}{2}-\frac{s}{4}+j$ & $\frac{a-1}{2}+\frac{s}{4}+j$ & $-a-s+2 j$ \\
\hline (c) & single cell & $\frac{a+1}{2}$ & $\frac{a-1}{2}+s-1$ & $-a-1$ \\
\hline (d) $\dagger$ & $j=0, \ldots, \frac{s}{4}-2$ & $\frac{a+1}{2}+1+j$ & $\frac{a-1}{2}+\frac{s}{2}+j$ & $-a-\frac{s}{2}+1+2 j$ \\
\hline (e) & single cell & $\frac{a+1}{2}+\frac{s}{4}$ & $\frac{a-1}{2}+\frac{s}{4}-1$ & $-a-\frac{s}{2}-1$ \\
\hline (f) $\dagger$ & $j=0, \ldots, \frac{s}{4}-2$ & $\frac{a+1}{2}+\frac{s}{4}+1+j$ & $\frac{a-1}{2}-\frac{s}{4}+j$ & $-a-s+1+2 j$ \\
\hline (g) $\dagger$ & $j=0, \ldots, \frac{s}{4}-2$ & $\frac{a+1}{2}+\frac{s}{2}+j$ & $\frac{a-1}{2}+j$ & $-a-\frac{s}{2}+2 j$ \\
\hline (h) & $j=0, \ldots, \frac{s}{4}-1$ & $\frac{a+1}{2}+\frac{3 s}{4}-1+j$ & $\frac{3 a+1}{2}+\frac{3 s}{4}+j$ & $\frac{s}{2}+2 j$ \\
\hline (i) & single cell & $\frac{a+1}{2}+s-1$ & $\frac{a-1}{2}-1$ & $-a-2$ \\
\hline (j) & $j=0, \ldots, \frac{a-1}{2}$ & $\frac{a+1}{2}+s+j$ & $\frac{3 a+1}{2}+s+j$ & $s+1+2 j$ \\
\hline (k) $*$ & $j=0, \ldots, \frac{a-1}{2}-$ & $a+s+1+j$ & $j$ & $-a+1+2 j$ \\
\hline (l) & $j=0, \ldots, \frac{s}{4}-1$ & $\frac{3 a+1}{2}+\frac{3 s}{4}+j$ & $\frac{a-1}{2}+\frac{3 s}{4}-$ & $\frac{s}{2}-1+2 j$ \\
\hline (m) & $j=0, \ldots, \frac{a-1}{2}$ & $\frac{3 a+1}{2}+s+j$ & $\frac{a-1}{2}+s+j$ & $s+2 j$
\end{tabular}

\section{Concluding remarks}

Our results improve the known lower bounds for the number of additive permutations, zero-sum arrays, some Skolem-type sequences, and some extremal Langford sequences. It seems highly likely that the bounds obtained in this paper apply to all pure, split, hooked and split-hooked Skolem sequences of sufficiently large orders. The recent paper [8] combines such bounds with graph labellings to generate Langford sequences. It seems likely that our new bounds can be combined with these techniques to generate improved estimates for the numbers of Langford sequences.

For small orders, the numbers of (pure) Skolem sequences and hooked Skolem sequences (and other related sequences) are tabulated in [4], while Table 8 of [7] gives the numbers of split Skolem (Rosa) sequences of orders $n \leq 12$. These numerical results strongly suggest that further improvements to our lower bounds are possible.

Since Skolem sequences may be used to construct solutions to Heffter's first and second difference problems, the bounds inform the numbers of these and of resulting cyclic Steiner triple systems. If improved bounds for $z_{2 t+1}$ are obtained in the future, these methods will lead to improved bounds for many related sequences. From Table 1, it will be seen that the ratio $z_{2 t+1} / z_{2 t-1}$ appears to increase with $t$, and to exceed $2 t$ for $t \geq 6$, strongly suggesting that $z_{2 t+1}>2^{t} t$ ! for all sufficiently large $t$. This is a weaker bound than might be suggested by Vardi's conjecture, but it is strongly supported by the computational evidence, and one might expect that most transversals are not $\mathbb{Z}$-transversals. 
Table 12: Subcase $2.4(r \equiv 3, s \equiv 2(\bmod 4))$, transversal in $R^{\prime}$.

\begin{tabular}{l|l|l|l|l} 
& Range & Row & Column & Entry \\
\hline \hline (a) & $j=0, \ldots, \frac{a}{2}-$ & $j$ & $a+s+j$ & $-a+2 j$ \\
& \multicolumn{1}{|c|}{$\frac{s-2}{4}-1$} & & & \\
\hline (b) & $j=0, \ldots, \frac{s-2}{4}-1$ & $\frac{a}{2}-\frac{s-2}{4}+j$ & $\frac{a}{2}+\frac{s-2}{4}+j$ & $-a-s+2 j$ \\
\hline (c) & single cell & $\frac{a}{2}$ & $\frac{a}{2}+s-1$ & $-a-1$ \\
\hline (d) & $j=0, \ldots, \frac{s-2}{4}-1$ & $\frac{a}{2}+1+j$ & $\frac{a}{2}+\frac{s}{2}-1+j$ & $-a-\frac{s}{2}+2 j$ \\
\hline (e) & single cell & $\frac{a}{2}+\frac{s-2}{4}+1$ & $\frac{a}{2}+\frac{s-2}{4}-1$ & $-a-\frac{s}{2}-1$ \\
\hline (f) & $j=0, \ldots, \frac{s-2}{4}-1$ & $\frac{a}{2}+\frac{s-2}{4}+2+j$ & $\frac{a}{2}-\frac{s-2}{4}-1+j$ & $-a-s+1+2 j$ \\
\hline (g) $\dagger$ & $j=0, \ldots, \frac{s-2}{4}-2$ & $\frac{a}{2}+\frac{s}{2}+1+j$ & $\frac{a}{2}+j$ & $-a-\frac{s}{2}+1+2 j$ \\
\hline (h) & $j=0, \ldots, \frac{s-2}{4}-1$ & $\frac{a}{2}+\frac{3 s-2}{4}+j$ & $\frac{3 a}{2}+\frac{3 s+2}{4}+j$ & $\frac{s}{2}+2 j$ \\
\hline (i) & single cell & $\frac{a}{2}+s-1$ & $\frac{a}{2}-1$ & $-a-2$ \\
\hline (j) & $j=0, \ldots, \frac{a}{2}$ & $\frac{a}{2}+s+j$ & $\frac{3 a}{2}+s+j$ & $s+2 j$ \\
\hline (k) $*$ & $j=0, \ldots, \frac{a}{2}-$ & $a+s+1+j$ & $j$ & $-a+1+2 j$ \\
\hline (l) & $j=0, \ldots, \frac{s-2}{4}$ & $\frac{3 a}{2}+\frac{3 s+2}{4}+j$ & $\frac{a}{2}+\frac{3 s-6}{4}+j$ & $\frac{s}{2}-1+2 j$ \\
\hline (m) & $j=0, \ldots, \frac{a}{2}-1$ & $\frac{3 a}{2}+s+1+j$ & $\frac{a}{2}+s+j$ & $s+1+2 j$ \\
\hline
\end{tabular}

\section{References}

[1] J. Abrham, Exponential lower bounds for the number of Skolem and extremal Langford sequences, Ars Combin. 22 (1986), 187-198.

[2] G. K. Bennett, M. J. Grannell and T. S. Griggs, Exponential lower bounds for the numbers of Skolem-type sequences, Ars Combin. 73 (2004), 101-106.

[3] N. J. Cavenagh and I. M. Wanless, On the number of transversals in Cayley tables of cyclic groups, Discrete Appl. Math. 158 (2010), 136-146, doi:10.1016/j.dam.2009.09.006.

[4] C. J. Colbourn and J. H. Dinitz (eds.), Handbook of Combinatorial Designs, Discrete Mathematics and its Applications, Chapman \& Hall/CRC, Boca Raton, Florida, 2nd edition, 2006, doi:10.1201/9781420010541, http: / /www. cems. uvm. edu/ jdinitz/hcd.html.

[5] C. J. Colbourn and A. Rosa, Triple Systems, Oxford Mathematical Monographs, Clarendon Press, New York, 1999.

[6] S. Eberhard, F. Manners and R. Mrazović, Additive triples of bijections, or the toroidal semiqueens problem, 2016, arXiv:1510.05987v3 [math.CO], submitted.

[7] M. Grüttmüller, R. Rees and N. Shalaby, Cyclically indecomposable triple systems that are decomposable, J. Combin. Math. Combin. Comput. 63 (2007), 103-122, http://www . combinatorialmath.ca/jcmcc/jcmcc63.html.

[8] S. C. López and F. A. Muntaner-Batle, Langford sequences and a product of digraphs, Eur. J. Combin. 53 (2016), 86-95, doi:10.1016/j.ejc.2015.11.004.

[9] N. J. A. Sloane (ed.), The On-Line Encyclopedia of Integer Sequences, published electronically at https://oeis.org. 
[10] I. Vardi, Computational Recreations in Mathematica, Addison-Wesley, Redwood City, California, 1991. 